\title{
Analytic continuation of scattering data as a method of obtaining characteristics of bound states
}

\author{
Leonid Blokhintsev $^{1 \mathrm{a}}$ and Dmitry Savin ${ }^{1}$ \\ ${ }^{1}$ Skobeltsyn Institute of Nuclear Physics, Lomonosov Moscow State University, Moscow, Russia
}

\begin{abstract}
The method of the analytic continuation of the effective range function is applied to obtain the asymptotic normalization coefficients for ${ }^{6} \mathrm{Li}$ nucleus in the $\alpha+d$ channel. Several sets of scattering phases obtained from the phase-shift analyses as well as from Faddeev calculations are used as an input. Since the $\alpha+d$ system possesses the low-lying inelastic threshold due to the dissociation of a deuteron, the approach used is generalized to include inelastic channels. The sensitivity of the obtained values of asymptotic normalization coefficients to the elastic channels coupling and to account of the inelastic channel is investigated.
\end{abstract}

\section{Introduction}

An asymptotic normalization coefficient (ANC) determines the asymptotics of a wave function of a nucleus $a$ in a binary channel $b+c$. ANCs are proportional to nuclear vertex constants (NVCs), which are on-shell matrix elements of the virtual processes $a \leftrightarrow b+c$. The NVC for the $a \leftrightarrow b+c$ vertex is expressed directly in terms of the residue of the elastic $b c$ scattering amplitude at the pole corresponding to the bound state $a$ [1]. ANCs and NVCs are fundamental nuclear characteristics. They are used actively in analyses of nuclear reactions within various approaches. The ANC for the channel $a \leftrightarrow b+c$ determines the probability of the configuration $b+c$ in nucleus $a$ at distances greater than the radius of nuclear interaction. Thus ANCs arise naturally in expressions for cross sections of nuclear reactions between charged particles at low energies, in particular, of astrophysical nuclear reactions. It was shown [2] that the cross section of the astrophysical $b(c, \gamma) a$ reaction with a good accuracy is determined by the value of the ANC in the $b+c$ channel. This conclusion made it possible to calculate astrophysical $S$ factors for a number of radiative capture processes.

ANC values could be determined in principle from microscopic calculations but such calculations are rather tedious. Theoretical results should be matched to the empirical ones obtained from data on scattering and reactions. One of the promising methods to extract ANCs is the analytic continuation of $b c$ scattering data to a pole of a scattering amplitude corresponding to a bound state $a$ and lying in the unphysical region of negative energies. The most effective way of realization of that procedure is the analytic continuation of the scattering (or effective range) function. This approach was employed in papers [3-5] for one-channel scattering to get the ANC and NVC values for several light nuclei. In papers [6] the method of the analytic continuation of the effective range function was generalized to the two coupled elastic channels what is important for the scattering of two particles with spins $1 / 2$ or spins 1 and 0 .

${ }^{\text {a }}$ Corresponding author : blokh@ srd.sinp.msu.ru

This is an Open Access article distributed under the terms of the Creative Commons Attribution License 2.0, which permits unrestricted use, distribution, and reproduction in any medium, provided the original work is properly cited. 
In the present work the method of the analytic continuation of the two-channel effective range function with account of the Coulomb interaction [6] is applied to obtain the ANCs and NVCs for ${ }^{6} \mathrm{Li}$ nucleus in the $\alpha+d$ channel.

\section{Methods and results}

The ANC values for the $\alpha+d$ system determine the cross section of the radiative capture ${ }^{4} \mathrm{He}(d, \gamma){ }^{6} \mathrm{Li}$, which is the only process of ${ }^{6} \mathrm{Li}$ formation in the Big Bang model. The available data on the values of the ANCs $C_{l}$ and the NVCs $G_{l}$ for that channel are characterized by a large spread, especially for $l=2$ $(l=0,2$ is the channel orbital angular momentum). The information on these data can be found in $[3,7]$ and in references therein. In the present approach several sets of scattering phases obtained from the phase-shift analyses of $d \alpha$ scattering [8,9] as well as from the Faddeev calculations [3] are used as the input. The coefficients of the Coulomb-modified two-channel effective range function [6] are found by fitting the input data including the binding energy $\epsilon$ of ${ }^{6} \mathrm{Li}$ in the $\alpha+d$ channel. The effective range function thus obtained is continued analytically to the pole corresponding to the ground state of ${ }^{6} \mathrm{Li}$.

Since the $\alpha+d$ system possesses the low-lying inelastic threshold due to the dissociation of a deuteron, the approach is generalized to include inelastic channels. Above the inelastic threshold the phase shifts $\delta_{l}$ and the mixing parameter $\varepsilon$, which are required to obtain parameters of the effective range function, become complex. Two methods of accounting for inelasticity effects in the effective range expansion with real parameters are used.

The first method consists just in ignoring imaginary parts of $\delta_{l}$ and $\varepsilon$. At that, inelasticity effects manifest themselves in violating pure elastic unitarity conditions.

The second method is more sophisticated and makes use of the approach proposed by Arndt and Roper [10] to parameterize the contribution of inelastic channels in the $N N$ scattering. Within this method, we utilize the values of $\delta_{l}$ and $\varepsilon$ from Refs. [3, 8,9] to construct the $S$ matrix for the $d \alpha$ scattering and the corresponding $K$ matrix. Above the inelastic threshold the $K$ matrix is complex. Then we use the real part of this $K$ matrix to construct the modified pure elastic $S$ matrix, which is expressed in terms of the modified values of the phase shifts and the inelasticity parameter. Although these modified values $\delta_{l}^{\prime}$ and $\varepsilon^{\prime}$ are real, they effectively take into account the presence of the inelastic channel.

The results of calculating ANCs $C_{l}$ and NVCs $G_{l}$ for ${ }^{6} \mathrm{Li}$ in the $\alpha+d$ channel are presented in Tables 1 and 2. The second lines in both Tables correspond to using the first method of treating the inelasticity effects and the third lines correspond to the second method.

Table 1. ANCs and NVCs calculated using the input from [3]

\begin{tabular}{|c|c|c|c|c|c|}
\hline Method & $\begin{array}{c}\text { Binding } \\
\text { energy } \boldsymbol{\epsilon}(\mathbf{M e V})\end{array}$ & $\boldsymbol{C}_{\mathbf{0}}, \mathbf{f m}^{\mathbf{- 1 / 2}}$ & $\boldsymbol{C}_{\mathbf{2}}, \mathbf{f m}^{\mathbf{- 1 / 2}}$ & $\boldsymbol{G}_{\mathbf{0}}{ }^{\mathbf{2}}, \mathbf{f m}$ & $\boldsymbol{G}_{\mathbf{2}}{ }^{\mathbf{2}}, \mathbf{f m}$ \\
\hline $1^{\text {st }}$ method & 2.41 & 2.03 & 0.0797 & 0.322 & 0.000496 \\
\hline $2^{\text {nd }}$ method & 2.41 & 1.97 & 0.0368 & 0.303 & 0.000106 \\
\hline
\end{tabular}

Table 2. ANCs and NVCs calculated using the input from $[8,9]$

\begin{tabular}{|c|c|c|c|c|c|}
\hline Method & $\begin{array}{c}\text { Binding } \\
\text { energy } \boldsymbol{\epsilon}(\mathbf{M e V})\end{array}$ & $\boldsymbol{C}_{\mathbf{0}}, \mathbf{f m}^{\mathbf{- 1 / 2}}$ & $\boldsymbol{C}_{\mathbf{2}}, \mathbf{f m}^{-\mathbf{1 / 2}}$ & $\boldsymbol{G}_{\mathbf{0}}{ }^{2}, \mathbf{f m}$ & $\boldsymbol{G}_{\mathbf{2}}{ }^{2}, \mathbf{f m}$ \\
\hline $1^{\text {st }}$ method & 1.47 & 1.96 & 0.0914 & 0.374 & 0.000364 \\
\hline $2^{\text {nd }}$ method & 1.47 & 1.88 & 0.0700 & 0.344 & 0.000106 \\
\hline
\end{tabular}


The values of $C_{l}$ and $G_{l}$ shown in Table 1 are found by utilizing the theoretical values of $\delta_{l}, \varepsilon$, and the binding energy $\epsilon$ of ${ }^{6} \mathrm{Li}$ in the $\alpha+d$ channel $(2.41 \mathrm{MeV})$. These values are calculated by solving the Faddeev equations for ${ }^{6} \mathrm{Li}$ in the three-body model $(n, p, \alpha)$ with neglecting the Coulomb interaction and using the simplified nucleon-nucleon interaction [3]. The quantities presented in Table 2 are obtained using the results of the energy-independent phase-shift analyses [7, 8] and the experimental value of $\epsilon(1.47 \mathrm{MeV})$.

\section{Discussion and Conclusions}

It is seen from Tables 1 and 2 that the values of the $s$ wave quantities $(l=0)$ for different inputs and different methods of taking account of inelasticity effects are rather close to each other (the spread of the $C_{0}$ values is about 3-6\%). These values are close to the value $C_{0}=2.28 \mathrm{fm}^{-1 / 2}$ cited in [7]. On the other hand, the $d$ wave quantities $(l=2)$ are sensitive both to the type of the input and to the way of allowing for inelasticity effects. This fact might be related to smaller absolute values and worse accuracy of the phase shifts $\delta_{2}$ from $[8,9]$ as compared with $\delta_{0}$. Nevertheless, in spite of large spread of the $C_{2}$ values presented in Tables 1 and 2, it is significant that in all cases the sign of $C_{2}$ (relative to $C_{0}$ ), which up to now has not been reliably established, turns out to be positive.

Comparing the values displayed in the second lines of Tables 1 and 2 with those shown in the third lines, one may conclude that using the method of Ref. [10] of accounting for inelasticity effects reduces the $C_{0}$ values by $3-4 \%$; at the same time the $C_{2}$ values change considerably.

Note that the $C_{0}$ values in Table 1 are obtained without account of the Coulomb interaction whereas those in Table 2, being based on the experimental data, include the Coulomb effects. The Coulomb parameter $\eta$ for ${ }^{6} \mathrm{Li}$ in the $\alpha+d$ channel is sizeable: $\eta=0.3$. Nonetheless, the $C_{0}$ values in Tables 1 and 2 are rather close to each other. This result is due to the fact that the ANC $C_{0}$ is affected in two compensating ways by the presence of the repulsive Coulomb interaction [11]: (1) The ANC decreases due to the decrease in binding energy; (2) the ANC increases due to the more rapid fall of the tail of the wave function. These two effects essentially cancel each other.

It is worthwhile to mention one more possible way to allow for inelasticity effects. Namely, if there is an inelastic threshold at $E=E_{0}$, then one may include into the effective range expansion an additional term which is complex at $E>E_{0}$. The explicit form of that term should lead to the correct analytic behavior of scattering amplitudes at the threshold $E=E_{0}$. According to the general theory of singularities of Feynman diagrams, a singular part of a scattering amplitude near a normal threshold behaves like $\left(E-E_{0}\right)^{(3 \mathrm{n}-5) / 2}$ at $n$ even and like $\left(E-E_{0}\right)^{(3 \mathrm{n}-5) / 2} \ln \left(E-E_{0}\right)$ at $n$ odd where $n=2,3,4, \ldots$ is a number of intermediate particles at the threshold. In the case of $d \alpha$ scattering the lowest inelastic threshold corresponds to the three-particle state $(n+p+\alpha)$, hence that additional term should be proportional to $\left(E-E_{0}\right)^{2} \ln \left(E-E_{0}\right)$. The coefficient multiplying that expression should be fitted to experimental data. The work on emloying this method is in progress.

In summary, we can say that employing the analytic continuation of the effective range expansion to determine the ANCs and NVCs for the ${ }^{6} \mathrm{Li} \rightarrow \alpha+d$ channel turns out to be successful. Nevertheless, the accuracy of the extracted quantities should be improved, especially for the $d$ state. To this end, it is desirable

- $\quad$ to measure more accurately the $d \alpha$ scattering differential cross section at low energies

- $\quad$ to perform the thorough phase-shift analysis of the corresponding data

- $\quad$ to perform Faddeev calculations of ${ }^{6} \mathrm{Li}$ in the 3-body model with realistic pair potentials and account of the Coulomb interaction

The work has been supported by the Russian Foundation for Basic Research under Grant No. 13-02-00399.

\section{References}

1. L.D. Blokhintsev, I. Borbeli, E.I. Dolinsky, Part. Nucl. 8, 485 (1977)

2. A.M. Mukhamedzhanov, R.E. Tribble, Phys. Rev. C 59, 3418 (1999) 
3. L.D. Blokhintsev, V.I. Kukulin, V.I. Sakharuk, D.A. Savin, E.V. Kuznetsova, Phys. Rev. C 48, 2390 (1993)

4. Yu.V. Orlov, B.F. Irgaziev, L.I. Nikitina, Phys. At. Nucl. 73, 757 (2010)

5. J.-M. Sparenberg, P. Capel, D. Baye, Phys. Rev. C 81, 011601 (2010)

6. L.D. Blokhintsev, Phys. At. Nucl. 74, 979 (2011); Bull. Russ. Acad. Sci. Physics 76, 425 (2012)

7. A.M. Mukhamedzhanov, L.D. Blokhintsev, B.F. Irgaziev, Phys. Rev. C 83, 055805, (2011)

8. B. Jenny, W. Grüebler, V. König, P.A. Schmelzbach, C. Schweizer, Nucl. Phys. A 397, 61 (1983)

9. W. Grüebler, P.A. Schmelzbach, V. König, R. Risler, D. Boerma, Nucl. Phys. A 242, 265 (1975)

10. R.A. Arndt, L.D. Roper, Phys. Rev. D 25, 2011 (1982)

11. D.R. Lehman, L.C. Maximon, J.L. Friar, Phys. Rev. C 37, 336 (1988) 